Gazi University
Journal of Science
PART A: ENGINEERING AND INNOVATION
http://dergipark.org.tr/gujsa

\title{
On Prime Ideals Related to an Ideal in a Commutative Ring
}

\author{
Ortaç ÖNEŞ ${ }^{1 *}$ ID
}

${ }^{1}$ Department of Mathematics, Akdeniz University, 07058 Antalya, Turkey

\begin{tabular}{ll}
\hline Keywords & Abstract \\
\hline Prime Ideal & This article focuses on the notion of prime ideal related to an ideal of a commutative ring. Some of its \\
characteristics are studied and the relations between ideals and prime ideals related to these ideals in a \\
commutative ring are examined. Then some useful connections among them are obtained. Both \\
Localization
\end{tabular}$\quad \begin{aligned} & \text { similarities and differences between prime ideals related to an ideal and prime ideals are pointed out. } \\
& \text { Moreover a direct connection between prime ideals related to an ideal and radical ideals is obtained. }\end{aligned}$

\begin{tabular}{lcc}
\hline Cite & \\
\hline Öneş, O. (2021). On Prime Ideals Related to an Ideal in a Commutative Ring. GU J Sci, Part A, 8(4), 451-458. \\
& \\
\hline Author ID (ORCID Number) & Article Process \\
\hline O. Öneş, 0000-0001-6777-9192 & Submission Date & 21.10 .2021 \\
& Revision Date & 17.11 .2021 \\
& Accepted Date & 29.11 .2021 \\
& Published Date & 01.12 .2021 \\
\hline
\end{tabular}

\section{INTRODUCTION}

In this article, all rings, which is denoted by $R$ are commutative with identity.

Historically, the discovery of the fact that every positive integer can be uniquely expressed as a product of prime powers arouse an analogous question in the context of ideal theory. Starting from mid $19^{\text {th }}$ century, works of mathematicians such as Kummer, Kronecker and Dedekind did much to shed light on this subject. These studies established a similar decomposability of ideals into prime ideals under certain conditions and as of today constitute a popular topic in algebraic number theory.

The concept of prime ideal is mostly at the center of studies in ring theory and many useful results related to prime ideals have been proved in Kirby (1966; 1969), McConnell \& Robson (1987), Jenkins \& Smith (1992), Lam (2001), Anderson \& Smith (2003), Naghipour (2005), Anderson \& Bataineh (2008), Lam \& Reyes (2008), Ebrahimpour \& Nekooei (2012), Öneş \& Alkan (2017; 2018; 2019a,b), Öneş (2020).

This article focuses on the relations between ideals and prime ideals related to these ideals in $R$.

The ideal $P \neq R$ is prime in $R$ if for any product $a b \in P$ of the elements $a, b$ of $R, a$ or $b$ is in $P$ (Atiyah $\&$ MacDonald, 1969).

The notion of primality of an ideal in $R$ was generalized in Öneş (2019) to the case in which $R$ is an ideal in general.

Definition 1.1. (Öneş, 2019) For any ideals $P, I$ in $R, P$ is $I$-prime if for any product $a b \in P$ of two elements $a, b$ in $I$, we have that 


$$
a \in P \text { or } b \in P
$$

Immediately, it can be seen that $R$-prime ideals coincides with prime ideals in $R$. When a specific ideal in $R$ is not mentioned, $*$-prime ideal is used instead of $I$-prime ideal.

Example 2.1 includes *-prime ideals in $R$. The connections between ideals and prime ideals related to them in $R$ are shown in Lemma 2.4 and Corollary 2.5. It is proved that the ideal quotient of $P$ by $I$ is $I$-prime while $P$ is $I$-prime ideal, in Lemma 2.6. When $P$ is maximal, its converse is also true. Moreover, in Proposition 2.7, which is stated in Öneş (2019), the proof that any epimorphism respects *-primality under a certain condition is given.

The radical of $I$, which is an ideal, in $R$ is defined as

$$
\left\{r \in R: \exists n \in \mathbb{Z}^{+}, r^{n} \in I\right\}=\sqrt{I}
$$

(Kaplansky, 1974). In Theorem 2.9, a connection between $\sqrt{I}$-prime and $I$-prime in $R$ is proved.

In Lemma 2.10, it is proved that in a local ring $R$, if $I$ is maximal and $P$ is $I$-prime, then $P$ is prime. A nonempty subset $S$ of $R$ is a multiplicative set if any product $a b$ of two elements $a, b \in S$ is also in $S$ (Matsumura, 1986).

In Theorem 2.11, the proof of *-primality in localization is given.

\section{PRIME IDEALS RELATED TO AN IDEAL}

\section{Example 2.1.}

i) Every prime ideal of $\mathbb{Z}$ is $I$-prime in $\mathbb{Z}$ for all ideals $I$ of $\mathbb{Z}$.

ii) $10 \mathbb{Z}$ and $15 \mathbb{Z}$ are not prime ideals in $\mathbb{Z}$ but $15 \mathbb{Z}$ is $10 \mathbb{Z}$-prime in $\mathbb{Z}$ and $10 \mathbb{Z}$ is $15 \mathbb{Z}$-prime in $\mathbb{Z}$.

\section{Proof.}

i) It is clear with Definition 1.1.

ii) It can be easily proved that $10 \mathbb{Z}$ and $15 \mathbb{Z}$ are not prime in $\mathbb{Z}$.

We show that $15 \mathbb{Z}$ is $10 \mathbb{Z}$-prime in $\mathbb{Z}$ and $10 \mathbb{Z}$ is $15 \mathbb{Z}$-prime in $\mathbb{Z}$.

Let $a, b \in 10 \mathbb{Z}$ such that $a b \in 15 \mathbb{Z}$. Then we have that

$$
a=10 x, b=10 y, 15 z=2^{2} 5^{2} x y, \text { where } x, y, z \in \mathbb{Z} \text {. }
$$

Hence it follows that $3 \mid x y$, meaning that $3 \mid x$ or $3 \mid y$.

If $3 \mid x$, then $a \in 30 \mathbb{Z} \subseteq 15 \mathbb{Z}$, meaning that $a \in 15 \mathbb{Z}$.

If $3 \mid y$, then $b \in 30 \mathbb{Z} \subseteq 15 \mathbb{Z}$, meaning that $b \in 15 \mathbb{Z}$.

Let $a, b \in 15 \mathbb{Z}$ such tha $\operatorname{tab} \in 10 \mathbb{Z}$. Then we have that

$$
a=15 x, b=15 y, 10 z=3^{2} 5^{2} x y, \text { where } x, y, z \in \mathbb{Z} \text {. }
$$


Hence it follows that $2 \mid x y$, meaning that $2 \mid x$ or $2 \mid y$.

If $2 \mid x$, then $a \in 30 \mathbb{Z} \subseteq 10 \mathbb{Z}$, meaning that $a \in 10 \mathbb{Z}$.

If $2 \mid y$, then $b \in 30 \mathbb{Z} \subseteq 10 \mathbb{Z}$, meaning that $b \in 10 \mathbb{Z}$.

Lemma 2.2. (Öneş, 2019) Let $I_{1}, I_{2}, P$ be ideals of $R$, where $I_{1} \subseteq I_{2}$, and let $P$ be $I_{2}$-prime in $R$. In this case, $P$ is $I_{1}$-prime in $R$.

Proof. Suppose $a, b \in I_{1}$ such that $a b \in P$.

Since $I_{1}$ is a subset of $I_{2}$, it follows that $a, b \in I_{2}$ with $a b \in P$. Because $P$ is $I_{2}$-prime in $R$, we have that

$$
a \in P \text { or } b \in P \text {, }
$$

which is the desired result.

The following corollary is obtained from Lemma 2.2.

Corollary 2.3. Let $I_{i}$ and $P$ be ideals of $R$ for every $i \in \Lambda$ and let $P$ be $I_{i}$-prime in $R$. Then

i) $P$ is $\bigcap_{i \in \Lambda} I_{i}$-prime in $R$.

ii) $P$ is $\prod_{i \in \Lambda} I_{i}$-prime in $R$, where $I_{i}$ is a co-maximal ideal of $R$ for every $i \in \Lambda$.

\section{Proof.}

i) Let $a, b \in \bigcap_{i \in \Lambda} I_{i}$ such that $a b \in P$, meaning that $a, b \in I_{i}$ for every $i \in \Lambda$.

By hypothesis, either $a \in P$ or $b \in P$ holds. Thus $P$ is $\bigcap_{i \in \Lambda} I_{i}$-prime in $R$.

ii) It is clear with (i).

Lemma 2.4. Let $P, I$ and $J$ be ideals of $R$. If $P$ is $I$-prime, then $\left(P:_{R} J\right)$ is $I$-prime.

Proof. Let $a, b \in I$ with $a b \in\left(P:_{R} J\right)$.

Assume that $b \notin\left(P:_{R} J\right)$.

Then we have $b j \notin P$ and $a b j \in P$ for an element $j$ in $J$. Since $a, b j \in I$ and $P$ is $I$-prime in $R$, we get that $a \in P$ or $b j \in P$. Because the element $b j$ is not in $P$, we have that

$$
a \in P \text {, meaning } a \in\left(P:_{R} J\right) \text {. }
$$

Thus $\left(P:_{R} J\right)$ is $I$-prime in $R$.

By using Lemma 2.2 and Lemma 2.4, the following result is obtained.

Corollary 2.5. Let $P, I$ and $J$ be ideals of $R$. If $P$ is $\left(I:_{R} J\right)$-prime, then $\left(P:_{R} J\right)$ is $I$-prime.

Proof. Let $P$ be $\left(I:_{R} J\right)$-prime.

Since $I \subseteq\left(I:_{R} J\right)$, then $P$ is $I$-prime by Lemma 2.2. Thus $\left(P:_{R} J\right)$ is $I$-prime with Lemma 2.4 . 
Lemma 2.6. Let $I, P$ be ideals in $R$, where $P$ is $I$-prime. Then $\left(P:_{R} I\right)$ is $I$-prime in $R$, where $I \neq P$. When $P$ is maximal, its converse is true.

Proof. Let $a, b \in I$ such that $a b \in\left(P:_{R} I\right)$.

Assume that $b \notin\left(P:_{R} I\right)$.

Then we have $b x \notin P$ and $a b x \in P$ for an element $x$ in $I$. Because $a, b x \in I$ and $P$ is $I$-prime in $R$, either $a \in P$ or $b x \in P$ holds. Since $b x \notin P$, we have that

$$
a \in P \text {, meaning } a \in\left(P:_{R} I\right) \text {. }
$$

Consequently $\left(P:_{R} I\right)$ is $I$-prime in $R$.

Suppose that $P$ is maximal in $R$ and $\left(P:_{R} I\right)$ is $I$-prime in $R$, where $I \neq P$.

Let $a, b \in I$ with $a b \in P$.

We have $a b I \subseteq P$, meaning $a b \in\left(P:_{R} I\right)$. Because $\left(P:_{R} I\right)$ is $I$-prime in $R$, it follows that $a \in\left(P:_{R} I\right)$ or $b \in\left(P:_{R} I\right)$. Since $P \subseteq\left(P:_{R} I\right)$, by the assumption it is true that

$$
a \in P \text { or } b \in P \text {. }
$$

Thus $P$ is $I$-prime in $R$.

Here, we prove the following proposition, which is stated in Öneş (2019).

Proposition 2.7. Let $S$ be a commutative ring with identity. Let $\varphi$ be an epimorphism from $R$ to $S$, let $I$ and $P$ be ideals in $R$ and $\operatorname{Ker} \varphi \subseteq P$. Then

$$
P \text { is } I \text {-prime iff } \varphi(P) \text { is } \varphi(I) \text {-prime. }
$$

Proof. $(: \Rightarrow)$ Let $a$ and $b$ be any elements of $\varphi(I)$ such that $a b \in \varphi(P)$.

Then we have $a=\varphi(x)$ and $b=\varphi(y)$ for $x, y \in I$. Then it follows that $a b=\varphi(x) \varphi(y)=\varphi(x y) \in \varphi(P)$. We also have $\varphi(x y)=\varphi(p)$ for $p \in P$. Since $\operatorname{Ker} \varphi \subseteq P$, we have $x y \in P$. Because $P$ is $I$-prime, we get that

$$
x \in P \text { or } y \in P \text {, meaning that } a \in \varphi(P) \text { or } b \in \varphi(P) .
$$

Thus $\varphi(P)$ is $\varphi(I)$-prime in $S$.

$(\Leftarrow$ : ) Let $a$ and $b$ be any elements of $I$ such that $a b \in P$.

Then we have $\varphi(a) \varphi(b)=\varphi(a b) \in \varphi(P)$ with $\varphi(a), \varphi(b) \in \varphi(I)$. Because $\varphi(P)$ is $\varphi(I)$-prime in $S$, either $\varphi(a) \in \varphi(P)$ or $\varphi(b) \in \varphi(P)$ holds. Since $\operatorname{Ker} \varphi \subseteq P$, it follows that

$$
a \in P \text { or } b \in P \text {. }
$$

Thus $P$ is $I$-prime in $R$. 
Proposition 2.8. Let $S$ be a commutative ring with identity. Let $\varphi$ be an epimorphism from $R$ to $S$, let $I$ be an ideal in $R$ and let $P$ be $\varphi(I)$-prime in $S$. Then $\varphi^{-1}(P)$ is $I$-prime in $R$.

Proof. Let $a$ and $b$ be any elements of $I$ such that $a b \in \varphi^{-1}(P)$.

Thus $\varphi(a b)=\varphi(a) \varphi(b)$ is in $P$, where $\varphi(a)$ and $\varphi(b)$ are in $\varphi(I)$. Because $P$ is $\varphi(I)$-prime ideal of $S$, we have that

$$
\varphi(a) \in P \text { or } \varphi(b) \in P \text {. }
$$

It follows that $a \in \varphi^{-1}(P)$ or $b \in \varphi^{-1}(P)$. Thus $\varphi^{-1}(P)$ is $I$-prime in $R$.

Theorem 2.9. Let $J, I$ be ideals of $R$.

i) $J$ is $\sqrt{I}$-prime in $R$.

ii) $J$ is $I$-prime in $R$.

iii) $\sqrt{J}$ is $I$-prime in $R$.

The above statement (i) implies statement (ii), which implies statement (iii). When $J=\sqrt{J}$, all three statements are equivalent.

\section{Proof.}

i $\Rightarrow$ ii) It is clear with Lemma 2.2.

ii $\Rightarrow$ iii) Suppose that $J$ is $I$-prime in $R$.

Let $a, b \in I$ such that $a b \in \sqrt{J}$.

Then we have that $(a b)^{n}=a^{n} b^{n} \in J$ for $n \in \mathbb{Z}^{+}$. We have also $a^{n}, b^{n} \in I$. Since $J$ is $I$-prime in $R$, either $a^{n} \in J$ or $b^{n} \in J$ holds. Thus we have that

$$
a \in \sqrt{J} \text { or } b \in \sqrt{J} \text {. }
$$

Consequently $\sqrt{J}$ is $I$-prime in $R$.

Let $J=\sqrt{J}$.

iii $\Rightarrow \mathbf{i})$ Suppose that $\sqrt{J}$ is $I$-prime in $R$.

Let $a, b \in \sqrt{I}$ such that $a b \in J$.

There exist positive integers $n, m$ such that $a^{n}, b^{m} \in I$. Thus we have that

$$
(a b)^{n m}=\left(a^{n}\right)^{m}\left(b^{m}\right)^{n} \in J=\sqrt{J} .
$$


Because $\sqrt{J}$ is $I$-prime in $R$, either $a \in \sqrt{J}$ or $b \in \sqrt{J}$ holds. Since $J=\sqrt{J}$, either $a \in J$ or $b \in J$ holds. Consequently $J$ is $\sqrt{I}$-prime in $R$.

Lemma 2.10. Let $I$ be a maximal ideal of a local ring $R$, let $P$ be a proper ideal of $R$ and let $P$ be $I$-prime in $R$. Then $P$ is prime in $R$.

Proof. Let $a, b \in R$ such that $a b \in P$.

We have the following three cases:

i) If $a$ and $b$ are invertible elements in $R$, then it follows that

$$
1_{R}=a^{-1} a b b^{-1} \in P \text {, meaning } P=R \text {. }
$$

It is a contradiction.

ii) If $a$ or $b$ is an invertible element in $R$, then we have that

$$
a \in P \text { or } b \in P \text {. }
$$

Consequently $P$ is prime in $R$.

iii) If $a$ and $b$ are not invertible elements in $R$, then we have that $a, b \in I$. Since $P$ is $I$-prime, we get that

$$
a \in P \text { or } b \in P \text {. }
$$

Consequently $P$ is prime in $R$.

Theorem 2.11. Let $S$ be a multiplicative subset of $R$ and let $I, P$ be ideals of $R$. If $P$ is an $I$-prime ideal of $R$, $I \cap S=\emptyset$ and $P \cap S=\emptyset$, then $S^{-1} P$ is a $S^{-1} I$-prime ideal of $S^{-1} R$.

Proof. Since $P \cap S=\emptyset, S^{-1} P$ is a proper ideal of $S^{-1} R$.

Let $\left(\frac{k}{s_{1}}\right),\left(\frac{l}{s_{2}}\right) \in S^{-1} I$ such that $\left(\frac{k}{s_{1}}\right)\left(\frac{l}{s_{2}}\right) \in S^{-1} P$.

There exist the elements $s_{3}, s_{4}, s_{5} \in S$ such that $k s_{3}, l s_{4} \in I$ and $k l s_{5} \in P$. Then we have that

$$
\left(k s_{3} s_{5}\right)\left(l s_{4}\right) \in P .
$$

Since $P$ is $I$-prime, either $\left(k s_{3} s_{5}\right) \in P$ or $\left(l s_{4}\right) \in P$ holds.

If $\left(k s_{3} s_{5}\right) \in P$, then it follows that $\left(\frac{k s_{3} s_{5}}{s_{1}}\right) \in S^{-1} P$. Thus we have that

$$
\left(\frac{1}{s_{3} s_{5}}\right)\left(\frac{k s_{3} s_{5}}{s_{1}}\right)=\left(\frac{k}{s_{1}}\right) \in S^{-1} P .
$$

If $\left(l s_{4}\right) \in P$, then it follows that $\left(\frac{l s_{4}}{s_{2}}\right) \in S^{-1} P$. Thus we have that 


$$
\left(\frac{1}{s_{4}}\right)\left(\frac{l s_{4}}{s_{2}}\right)=\left(\frac{l}{s_{2}}\right) \in S^{-1} P
$$

Consequently $S^{-1} P$ is a $S^{-1} I$-prime ideal of $S^{-1} R$.

\section{ACKNOWLEDGEMENT}

This study is supported by the Scientific Research Project Administration of Akdeniz University with the research project number FBA-2019-4969.

The author thanks the referees for their valuable suggestions and comments.

\section{CONFLICT OF INTEREST}

The author declares no conflict of interest.

\section{REFERENCES}

Anderson, D. D., \& Bataineh, M. (2008). Generalizations of prime ideals. Communications in Algebra, 36, 686-696.

Anderson, D. D., \& Smith, E. (2003). Weakly prime ideals. Houston Journal of Mathematics, 29, 831-840.

Atiyah, M. F., \& MacDonald, I. G. (1969). Introduction to Commutative Algebra. Addison-Wesley.

Ebrahimpour, M., \& Nekooei, R. (2012). On generalizations of prime ideals. Communications in Algebra, 40, 1268-1279.

Jenkins, J., \& Smith, P.F. (1992). On the prime radical of a module over a commutative ring. Communications in Algebra, 20, 3593-3602.

Kaplansky, I. (1974). Commutative Rings. The University of Chicago.

Kirby, D. (1966). Components of ideals in a commutative ring. Ann. Mat. Pura Appl., 4, 109-125.

Kirby, D. (1969). Closure operations on ideals and submodules. J. London. Math. Soc., 44, 283-291.

Lam, T. Y. (2001). A First Course in Noncommutative rings. Springer.

Lam, T. Y., \& Reyes, M. L. (2008). A prime ideal principle in commutative algebra. Journal of Algebra, 319 , 3006-3027.

Matsumura, H. (1986). Commutative Ring Theory. Cambridge University Press.

McConnell, J. C., \& Robson, J. C. (1987). Noncommutative Noetherian Rings. Wiley Chichester.

Naghipour, A. R. (2005). A simple proof of Cohen's theorem. The American Mathematical Monthly, 112, 825826.

Öneş, O. (2019). A generalization of prime ideals in a commutative ring. In: Proceedings Book of the 2nd Mediterranean International Conference of Pure Applied Mathematics and Related Areas, Paris, France, 2831 August, 124-126.

Öneş, O. (2020). On radical formula in modules over noncommutative rings. Filomat, 34(2), 443-449.

Öneş, O., \& Alkan, M. (2017). On the left O-prime ideals over a noncommutative ring. Advanced Studies in Contemporary Mathematics, 27(1), 107-113.

Öneş, O., \& Alkan, M. (2018). A note on graded ring with prime spectrum. Advanced Studies in Contemporary Mathematics, 28(4), 625-634. 
Öneş, O., \& Alkan, M. (2019a). Multiplication modules with prime spectrum. Turkish Journal of Mathematics, 43(4), 2000-2009.

Öneş, O., \& Alkan, M. (2019b). Zariski subspace topologies on ideals. Hacettepe Journal of Mathematics and Statistics, 48(6), 1667-1674. 